\title{
Avaliação dos serviços de saúde bucal na atenção primária à saúde: perspectivas regionais com base no PMAQ
}

\section{Evaluation of oral health services in primary health care: regional perspectives based on PMAQ}

\section{Evaluación de los servicios de salud bucal en la atención primaria de salud: perspectivas regionales basadas en PMAQ}

\author{
Orlando Luiz do Amaral Júnior ${ }^{1}$ \\ Maria Laura Braccini Fagundes ${ }^{1}$ \\ Gabriele Rissotto Menegazzo \\ Luisa Helena do Nascimento Tôrres \\ Jessye Melgarejo do Amaral Giordani ${ }^{1}$
}

RESUMO: Objetivo:O objetivo do estudo foi analisar o perfil epidemiológico da assistência odontológica dos municípios da $4^{\mathrm{a}}$ Coordenadoria Regional de Saúde do Rio Grande do Sul (4 ${ }^{\mathrm{a}}$ CRS/RS) a partir dos dados gerados no $2^{\text {a }}$ ciclo do Programa Nacional de Melhoria do Acesso e da Qualidade da Atenção Básica (PMAQ-AB). Metodologia:Trata-se de um estudo observacional transversal utilizando como instrumento os microdados da avaliação externa do PMAQ-AB divididos nas dimensões de estrutura das unidades básicas, processo de trabalho das equipes de atenção à saúde bucal e usuários do serviço. Dos 32 municípios pertencentes à $4^{\mathrm{a}} \mathrm{CRS} / \mathrm{RS}, 21$ aderiram ao programa e fazem parte deste estudo, sendo que estes sãodivididos em duas regiões de saúde. Esse estudo foi aprovado pelo Comitê de Ética em Pesquisa da Universidade Federal de Santa Maria e a análise estatística dos dados foi realizada através do programa Stata 14.0, onde foram realizadas análises de frequência para variáveis selecionadas. Resultados:Participaram da avaliação 56 Unidades de Atenção Básica, 44 equipes de saúde bucal e 261 usuários do serviço de atenção básica e com isso observamos que, apesar da maioria das unidades possuírem atendimento odontológico com agendamento e capacidade de resolução dos casos, a maioria dos usuários não o utilizam. Conclusão:Com estes resultados almejamos desencadear processos que tragam

1 Programa de Pós-Graduação em Ciências Odontológicas, Universidade Federal de Santa Maria, Santa Maria/RS, Brasil.

ISSN 1982-8829 Tempus, actas de saúde colet, Brasília, 14(1), 143-159 mar, 2020. Epub Mai/2020 
melhorias para as unidades, equipes e usuários da atenção básica no que tange o planejamento, organização e cuidado em saúde e concluímos que este tipo de avaliação se mostra importante para o desenvolvimento de ações de saúde pública.

Palavras chave: Assistência odontológica, Atenção primária à saúde,Avaliação em saúde.

\begin{abstract}
Objective: The aim of this study was to analyze the epidemiological profile of dental attendance of the municipalities of the 4th Regional Health Coordination of Rio Grande do Sul (4th CRS/RS) from the data generated in the 2nd cycle of the National Program for Improving Access and Quality in Primary Care (PMAQ-AB). Methods: This is an observational crosssectional study using microdata of the external evaluation of PMAQ-AB divided into the structure dimensions of the basic units, work process of the oral health care teams and service users. Of the 32 municipalities belonging to the 4th CRS/RS, 21 joined the program and are part of this study, and these are divided into two health regions. This study was approved by the Research Ethics Committee of the Federal University of Santa Maria and the statistical analysis of the data was performed using the software Stata 14.0, where frequency analyzes were performed for selected variables. Results: Participated in this evaluation 56 Primary Care Units, 44 oral health teams and 261 users of the primary care service. Thus, despite the fact that most units have dental care with programming and resolution of cases, most users do not use it. Conclusion: With these results we intention to provoke processes that bring improvements to primary care units, teams and users regarding planning, organization and health care and we conclude that this type of evaluation is important for the development of public health actions.
\end{abstract}

Keywords: Dental care,Health evaluation,Primary health care.

RESUMEN: Objetivo: El objetivo del estudio fue analizar el perfil epidemiológico de la atención odontológica de los municipios de la 4ta Coordinación Regional de Salud de Rio Grande do Sul (4to CRS/RS) a partir de los datos generados en el 2do ciclo del Programa Nacional de Mejora del Acceso y Calidad de la Atención Primaria (PMAQ-AB). Metodología: Este es un estudio observacional transversal de evaluación externa de PMAQ-AB divididos en las dimensiones de la estructura de las unidades básicas, el proceso de trabajo de atención de la salud bucal y los usuarios del servicio. De los 32 municipios que pertenecen al 4to CRS/RS, 21 se unieron al programa. Este estudio fue aprobado por el Comité de Ética en Investigación de la Universidad Federal de Santa María y el análisis estadístico de los datos se realizó utilizando el programa Stata 14.0, donde se realizaron análisis de frecuencia para variables seleccionadas. Resultados: Participaron en la evaluación 56 Unidades de Atención Primaria, 44 equipos de salud bucal y 261 usuarios del servicio, a pesar de que la mayoría de las unidades tienen atención dental con programación y resolución de casos, la mayoría de los usuarios no lo usan. Conclusión: Con estos resultados, nuestro objetivo es desencadenar procesos que traigan mejoras a las unidades de atención primaria, los equipos y los usuarios con respecto a la planificación, la organización y la atención médica y odontológica y concluimos que este tipo de evaluación es importante para el desarrollo de acciones de salud pública. 
Contraseñas: Atención odontológica, Atención primaria de salud, Evaluación em salud.

\section{INTRODUÇÃO}

A saúde bucal ao longo da história brasileira esteve inserida em um contexto de modelos de saúde assistenciais e excludentes, assim prevalecendo práticas tecnicistas, biologicistas e curativistas(1). Com o advento do Sistema Único de Saúde (SUS), avançou-se na assistência social e política, redefinindo as atribuições de cada nível governamental e criando novos espaços de participação social (2). Logo, o modelo brasileiro de atenção à saúde tem se caracterizado pela união de ações e serviços de promoção, recuperação e proteção da saúde, sendo organizado de forma regionalizada complementando a lógica de planejamento integrado. Compreende noções de territorialidade, não se tornando restrito à abrangência municipal, mas respeitando seus limites como unidade indivisível, permitindo otimizar os recursos disponíveis (3). Também éhierarquizado, tendo a Atenção Primária à Saúdecomo principal porta de entrada e ordenadora dos serviços (3).

Com o intuito de fortalecer a APS, o Ministério da Saúde criou, a partir da portaria $\mathrm{n}^{\mathrm{o}} 1.654$ de 19 de julho de 2011, o Programa de Melhoria do Acesso e Qualidade da Atenção Básica (PMAQ$\mathrm{AB})$ que visa ampliar o acesso da Atenção Básica $(\mathrm{AB})$ e contribuir para geração de um padrão de qualidade comparável em todo o país (4). Busca-se, através deste programa, o fortalecimento da gestão da $\mathrm{AB}$, a qual deve incentivar e promover a autoavaliação e a educação permanente dos profissionais, favorecendo o aprimoramento da atenção à saúde e implementando políticas, com perspectiva de qualificar a $\mathrm{AB}$ (5), havendo também um incentivo financeiro para os municípios que atingirem melhorias no padrão de qualidade das equipes de saúde. O PMAQ-AB situa a avaliação dos serviços de saúde como estratégia permanente para a tomada de decisão e ação central para a melhoria do modelo vigente que rege as ações de saúde, sendo esta considerada um atributo fundamental a ser alcançado no SUS (6).

A avaliação dos serviços tem como objetivo subsidiar a tomada de decisão de gestores e formuladores de políticas, e consiste em fazer um julgamento de valor sobre determinada intervenção, o qual possa se traduzir em ações de transformação e melhoria da mesma (7). A avaliação normativa considera a existência de uma forte relação entre o respeito aos critérios as normas estabelecidas e os resultados da intervenção. Segundo Donabedian informações da qualidade dos cuidados podem ser divididas em três categorias: Estrutura, Processo e Resultados. O eixo Estrutura responsável por englobar questões relacionadas a recursos humanos, estrutura física, financiamento e equipamento. $\mathrm{O}$ eixo Processo envolve as questões de diagnóstico, prevenção e educação às pessoas. E o eixo Resultados diz respeito aos efeitos dos cuidados sobre as pessoas e populações(8). Essas categorias são muito utilizadas como base para avaliações, as quais devem ser incentivadas como práticas regulares nos serviços de saúde (8).

A partir da avaliação do PMAQ-AB, os resultados obtidos em um estudo de Cassoti (2014) com relação as equipes de saúde bucal brasileiras, considerando todas as macrorregiões, têm 
demonstrado que $45 \%$ dos usuários têm dificuldade de acesso à consulta odontológica e, quando a obtêm, existem empecilhos na prestação da garantia de continuidade do tratamento nas regiões Norte e Nordeste do Brasil (9). Um estudo realizado em uma região da Rede Regional de Atenção à Saúde do Estado de São Paulo, composta pelos Departamentos Regionais de Saúde das cidades de Barretos, Araraquara, Franca e Ribeirão Preto, envolveu 90 municípios e uma população total de 3.307.320 habitantes(10)onde se observou que somente $29 \%$ dos usuários referiram conseguir marcar atendimento com o cirurgião-dentista na sua unidade de saúdede referência (11). Com isso, pode-se observar que ainda são escassas as produções no que se refere aos estudos de avaliação da qualidade da atenção odontológica no Brasil (12), embora os resultados já obtidos apontam que as políticas de saúde bucal possuem lacunas tanto na oferta de acesso quanto na organização e qualidade do processo de trabalho(12).

Considerando que as produções destas informações são estratégicas para a gestão do SUS, com destaque para os resultados de avaliações bem estruturadas e contínuas e sua importânciapara o fortalecimento da Rede de Atenção à Saúde, é necessário que se produza e divulgue resultados referentes às mesmas. Ainda visto que essas informações geradas através do $2^{\circ}$ Ciclo do PMAQ$\mathrm{AB}$ são incipientes em relação à assistência odontológica na região Sul do país, tomou-se como objetivo deste estudo descrever alguns indicadores de qualidade dos serviços de saúde bucal de municípios pertencentes a $4^{\circ}$ Coordenadoria Regional de Saúde do sul do Brasil.

\section{METODOLOGIA}

Trata-se de um estudo observacional transversal que avaliou 21 municípios dos 32 que constituem a $4^{\mathrm{a}}$ Coordenadoria Regional de Saúde do estado do Rio Grande do Sul (4 CRS/RS)considerando o $2^{\circ}$ ciclo do PMAQ-AB.

A $4^{\text {a }}$ CRS/RS possui uma população de 541.247 habitantes e 32 municípios, divididos em duas regiões de saúde: Verdes Campos e Entre Rios, conforme observa-se na Figura 1. A 4 a CRS/RS tem como sede a cidade de Santa Maria, localizada a $286 \mathrm{~km}$ da capital do estado do RS (13). A região Verdes Campos possui uma população de 438.318 habitantes, com 21 municípiose cobertura de APS de 57,9\%. Embora $81,7 \%$ da população viva na zona urbana e $18,3 \%$ na zona rural, a principal fonte de renda dos municípios advém da agropecuária eo Índice de Desenvolvimento Humano (IDH) da região é de 0,703 (Médio)com uma taxa de analfabetismo de 4,5\% (13). Com relação a região Entre Rios, a mesma é composta por 127.285 habitantes em 11 municípios e cobertura da APS de $85,9 \%$. A maioria da população que reside nesta região vive em zona urbana também e o IDH dela é 0,687 (Médio)com 31,7\% da população possuindo renda mensal inferior a um terço do salário mínimo brasileiro(13). 
Figura 1 - Mapa da $4^{\text {a }}$ Coordenadoria Regional de Saúde do estado do Rio Grande do Sul e seus municípios pertencentes de acordo com suas regiões de saúde.

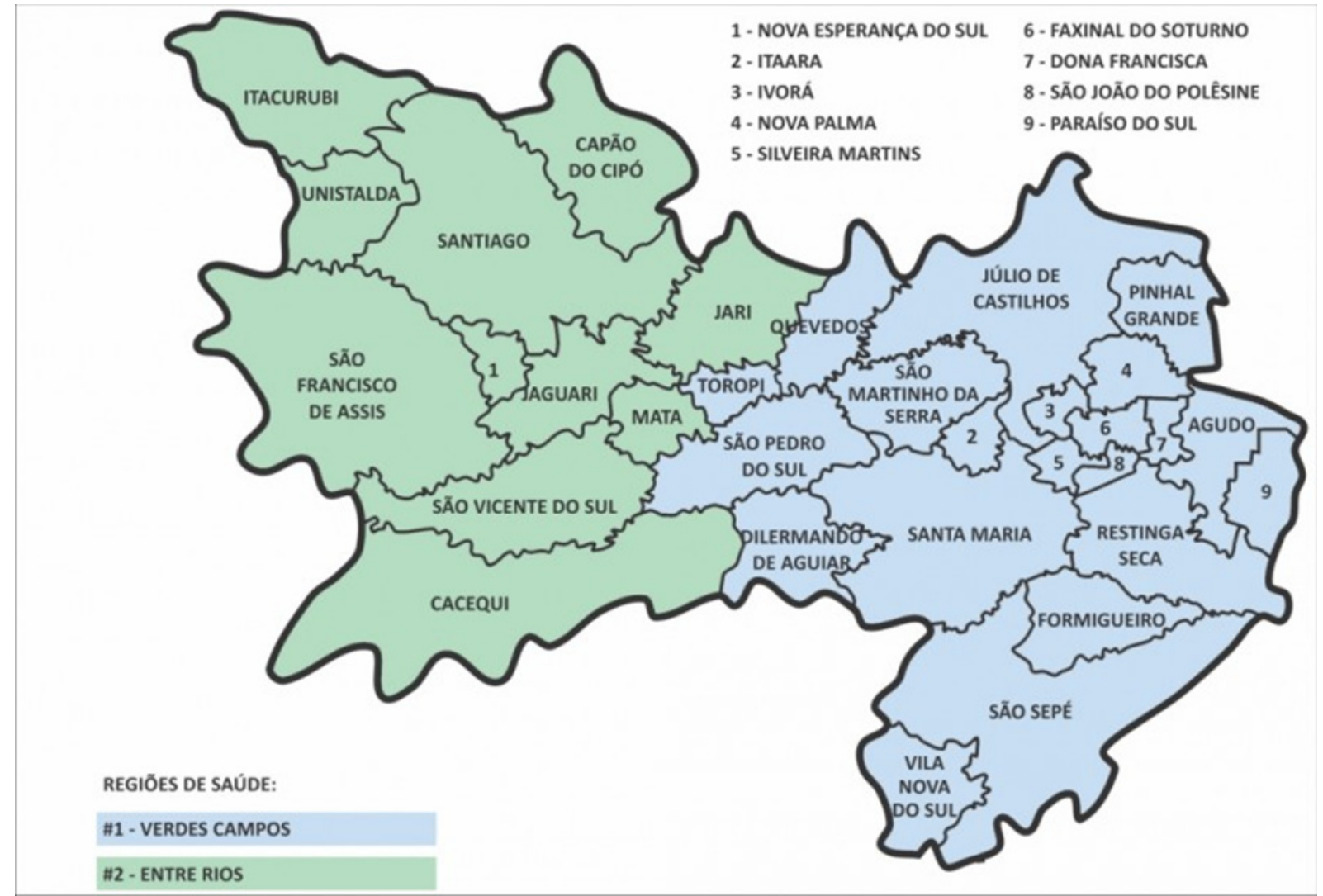

Fonte: Resoluçãon ${ }^{\circ}$ 518/17 - Secretaria de Saúde/RS (2017).

Foram utilizados neste estudo os microdados da avaliação externa do PMAQ-AB,os quais são públicos e encontram-se disponíveis no site do programa(aps.saude.gov.br/ape/pmaq/ciclo2). Estas informações correspondem ao $2^{\circ}$ ciclo do PMAQ, e estão subdivididas em três módulos: observação na UBS; entrevista com o profissional da equipe de Atenção Básica com verificação de documentos; e entrevista com usuários na UBS. A seleção dos participantes se deu por amostra de conveniência, envolvendo os participantes disponíveis para tal estudo, ou seja, aqueles que se encontravam disponíveis nas Unidades Básicas de Saúde que aceitaram aderir ao programa nos momentos de coleta.

A avaliação externa foi realizada através da aplicação de um questionário estruturadocontendo informações sobreas condições de acesso e qualidade das equipes participantes. Assim, entrevistadores previamente treinados visitaram as equipes da APS, após contato com a gestão municipal, conforme itinerário planejado pelas instituições e coletaram os dados através do uso de tablets contendo as questões. As avaliações, a fim de preservar o anonimato e priorizar a privacidade dos entrevistados, foram realizadas em locais reservados.

As variáveis do estudo foram selecionadas e extraídas da avaliação por tratarem-se de questões fundamentais da APS e, por serem oriundas dos três módulos da avaliação externa, foram organizadas de acordo com a abordagem para avaliação da qualidade dos serviços de saúde de Donabedian(8): 
i) estrutura das UBS;ii) processo de trabalho das equipes de saúde bucal;iii) resultados reportados pelos usuários do serviço.

A dimensão estrutural foi composta por três variáveis, referente às UBS: possuir consultório odontológico, cirurgião dentista e auxiliar de saúde bucal. A dimensão de processo foi composta por variáveis que questionaram os profissionais com relação a possuir formação complementar, realizar atividade de planejamento de suas ações mensais eter disponíveis informações que auxiliam na análise de situação de saúde por parte da gestão. Também foi verificado a rotina das equipes de saúde bucal em participardas reuniões de equipe da atenção básica, receber apoio de outros núcleos profissionais para auxiliar na resolução de casos considerados complexos, assim como suaforma de agendamento das consultas odontológicas, existência de atendimento especializado, realização de ações de prevenção do câncer bucal, acompanhamento de gestantes, atendimento a crianças menores de 5 anos de idade, visitas domiciliares, atividades em escolas e creches e oferta de serviços de próteses dentária.A última dimensão, dos resultados reportados pelo usuário, compreendeu variáveis referentes às questões que verificaram se os mesmos já haviam consultado com o dentista na unidade de saúde. Daqueles que o fizeram, buscou-se saber qual a opinião deles quanto ao horário do atendimento clínico odontológico; se são escutados quando procuram o atendimento mesmo sem hora marcada; como são recebidos pela equipe de saúde bucal; se acreditam que as orientações recebidas atendem às suas necessidades; se avaliam o tempo da consulta como sendo suficiente; e questões quanto às condições de limpeza e uso dos equipamentos odontológicos.

A análise estatística dos dados foi realizada através do programa estatístico Stata 14.0(StataCorporation, CollegeStation, TX, USA), onde foram realizadas análises de frequência absoluta e relativa para as variáveis selecionadas de cada dimensão de acordo com a região de saúde. Embora os microdados da avaliação externa no PMAQ, o protocolo de pesquisa foi submetido e aprovado pelo Comitê de Ética em Pesquisa da Universidade Federal de Santa Maria sob número do parecer 2.887 .912 e autorizado pela $4^{\text {a }} \mathrm{CRS} / \mathrm{RS}$.

\section{RESULTADOS}

Dos 32 municípios pertencentes à $4^{\mathrm{a}} \mathrm{CRS} / \mathrm{RS}, 21$ aderiram ao programa e fazem parte deste estudo, sendo que destes, 13 pertenciam à região Verdes Campos e 8 à região Entre Rios. Participaram da avaliação 56 Unidades de Atenção Básica, 44 equipes de saúde bucal e 261 usuários do serviço de atenção básica.

Os dados das variáveis referentes à estrutura das UBS de acordo com as regiões de saúde da $4^{\mathrm{a}} \mathrm{CRS} / \mathrm{RS}$ encontram-se na Tabela 1 . Das 56 unidades analisadas, 71,4\% possuíam consultório odontológico, sendo destas, 23 (69,7\%) na região Verdes Campos e 17 (73,9\%) na região Entre Rios.Do total das UBS avaliadas, 25\% não possuíam cirurgião dentista e 33,9\% não possuía auxiliares de saúde bucal, sendo que, das que o possuíam, $62,5 \%$ e 58,9\% contava com o auxílio de apenas um profissional cirurgião dentista e auxiliar de saúde bucal, respectivamente. 
Tabela 1 -Número e proporção de Unidades Básicas de Saúde participantes do PMAQ de acordo com a região de saúde, $4^{\mathrm{a}} \mathrm{CRS} / \mathrm{RS}$, em 2015.

\begin{tabular}{l|c|c|c} 
Variáveis* & $\begin{array}{c}\text { Região } \\
\text { Verdes } \\
\text { Campos }\end{array}$ & $\begin{array}{c}\text { Região } \\
\text { Entre Rios } \\
\mathbf{n}(\mathbf{\%})\end{array}$ & $\begin{array}{c}\text { TOTAL } \\
\text { n (\%) }\end{array}$ \\
Presença de consultório odontológico na unidade & $23(69,7)$ & $17(73,9)$ & $40(71,4)$ \\
Não possui cirurgião dentista na unidade & $10(30,3)$ & $4(17,4)$ & $14(25,0)$ \\
Possui apenas um cirurgião dentista na unidade & $19(57,6)$ & $16(69,6)$ & $35(62,5)$ \\
Não possui auxiliar de saúde bucal na unidade & $15(45,4)$ & $4(17,4)$ & $19(33,9)$ \\
Possui apenas um auxiliar de saúde bucal na unidade & $16(48,5)$ & $17(73,9)$ & $33(58,9)$ \\
\hline
\end{tabular}

* Dados referentes ao módulo I da avaliação externa.

Na tabela 2 são apresentadas as características das regiões de saúde em relação ao processo de trabalho das equipes de saúde bucal entrevistadas. Dentre os profissionais de equipe de saúde bucal que responderam aos questionamentos, $79,6 \%$ possuíam formação complementar, 80,8\% na região Verdes Campos e 77,8\% na região Entre Rios e mais de 88\% relataram realizar mensalmente atividades de planejamento e programação de suas ações. A maioria dos entrevistados relatou receber da gestão informações que auxiliam na análise da situação de saúde (75\%) e receber apoio de outros núcleos profissionais para a resolução de casos complexos $(63,6 \%)$, apesar deste percentual ser maior na região Entre Rios, porém, 47,7\% relataram haver ofertado em sua rede de atenção odontológica algum atendimento especializado. As equipes de saúde bucal em sua grande maioria participam das reuniões de equipe da atenção básica, promovem ações de prevenção e detecção do câncer de boca, disponibilizam acompanhamento para gestantes e crianças pré-escolares e realizam visitas domiciliares e atividades nas escolas e creches, mas cerca de $81 \%$ não tem como ofertar aos seus usuários reabilitações protéticas. Em relação ao agendamento das atividades clínicas, a grande maioria das equipes o faz através de agenda e atendimento de demanda espontânea simultaneamente e garante o retorno para continuidade do tratamento ( $88,6 \%$ e $90,9 \%$, respectivamente). 
Tabela 2=Número e proporção das equipes de saúde bucal participantes do PMAQ de acordo com a região de saúde, $4^{\mathrm{a}} \mathrm{CRS} / \mathrm{RS}$, em 2015.

\begin{tabular}{|c|c|c|c|}
\hline Variáveis* & Campos & $\begin{array}{l}\text { Entre Rios } \\
\text { n (\%) }\end{array}$ & $\begin{array}{l}\text { TOTAL } \\
\text { n (\%) }\end{array}$ \\
\hline $\begin{array}{l}\text { Profissional respondente tem formação } \\
\text { complementar }\end{array}$ & $21(80,8)$ & $14(77,8)$ & $35(79,6)$ \\
\hline $\begin{array}{l}\text { Há mensalmente atividade de planejamento e } \\
\text { programação de ações }\end{array}$ & $23(88,5)$ & $16(88,9)$ & $39(88,6)$ \\
\hline $\begin{array}{l}\text { A gestão disponibiliza informações que auxiliam } \\
\text { na análise da situação de saúde }\end{array}$ & $18(69,2)$ & $15(83,3)$ & $33(75,0)$ \\
\hline $\begin{array}{l}\text { A equipe participa de reuniões de equipe da atenção } \\
\text { básica }\end{array}$ & $22(84,6)$ & $17(94,4)$ & $39(88,6)$ \\
\hline $\begin{array}{l}\text { A equipe recebe apoio de outros núcleos } \\
\text { profissionais para a resolução de casos complexos }\end{array}$ & $15(57,7)$ & $13(72,2)$ & $28(63,6)$ \\
\hline $\begin{array}{l}\text { O atendimento clínico garante consultas } \\
\text { por agendamento e demanda espontânea } \\
\text { simultaneamente }\end{array}$ & $24(92,3)$ & $15(83,3)$ & $39(88,6)$ \\
\hline $\begin{array}{l}\text { A equipe garante agendamento de retorno para } \\
\text { continuidade do tratamento }\end{array}$ & $23(88,5)$ & $17(94,4)$ & $40(90,9)$ \\
\hline $\begin{array}{l}\text { É ofertado na rede de atenção odontológica algum } \\
\text { atendimento especializado }\end{array}$ & $12(46,2)$ & $9(50,0)$ & $21(47,7)$ \\
\hline $\begin{array}{l}\text { A equipe realiza ações de prevenção e detecção do } \\
\text { câncer de boca }\end{array}$ & $21(80,8)$ & $14(77,8)$ & $35(79,6)$ \\
\hline A equipe realiza acompanhamento de gestantes & $23(88,5)$ & $17(94,4)$ & $40(90,9)$ \\
\hline $\begin{array}{l}\text { A equipe disponibiliza atendimento para crianças } \\
\text { de até } 5 \text { anos de idade }\end{array}$ & $23(88,5)$ & $18(100)$ & $41(93,2)$ \\
\hline A equipe realiza visita domiciliar & $23(88,5)$ & $16(88,9)$ & $39(88,6)$ \\
\hline Não há oferta de próteses dentárias no município & $21(80,8)$ & $15(83,3)$ & $36(81,8)$ \\
\hline A equipe realiza atividades nas escolas e creches & $24(92,3)$ & $18(100)$ & $42(95,4)$ \\
\hline
\end{tabular}

* Dados referentes ao módulo II da avaliação externa.

Em relação à dimensão resultados, de um total de 261 usuários do serviço de atenção primária da região, apenas $101(38,7 \%)$ relataram haver passado por alguma consulta odontológica naquele local e mais de $\mathbf{8 8} \%$ julgaram o horário de atendimento odontológico suficiente para atender às suas necessidades. Dos usuários que realizaram atendimento com a equipe de saúde bucal, cerca da metade relatou ser escutado por ela mesmo quando procurava o serviço sem horário marcado, mais de $78 \%$ se disseram satisfeitos com o serviço prestado e a grande maioria consideraram as orientações passadas pela equipe suficientes para suas necessidades além do tempo de atendimento suficiente. No que diz respeito às condições dos consultórios odontológicos, os usuários relataram boas condições de limpeza (98\%) e boas condições de uso $(92,1 \%)$ (tabela 3). 
Tabela 3 - Número e proporção de usuários dos serviços de saúde bucal participantes do PMAQ de acordo com a região de saúde, $4^{\mathrm{a}} \mathrm{CRS} / \mathrm{RS}$, em 2015.

\begin{tabular}{|c|c|c|c|}
\hline Variáveis* & $\begin{array}{c}\text { Região Verdes } \\
\text { Campos } \\
\text { n (\%) }\end{array}$ & $\begin{array}{c}\text { Região } \\
\text { Entre Rios } \\
\text { n }(\%)\end{array}$ & $\begin{array}{l}\text { TOTAL } \\
\text { n (\%) }\end{array}$ \\
\hline $\begin{array}{l}\text { Usuário respondente que passou por consulta } \\
\text { odontológica }\end{array}$ & $59(34,7)$ & $42(46,2)$ & $101(38,7)$ \\
\hline $\begin{array}{l}\text { O horário de atendimento odontológico atende } \\
\text { às necessidades }\end{array}$ & $52(89,7)$ & $34(87,2)$ & $86(88,7)$ \\
\hline $\begin{array}{l}\text { O usuário é escutado pela equipe de saúde bucal } \\
\text { mesmo sem hora marcada }\end{array}$ & $30(50,9)$ & $23(54,8)$ & $53(52,5)$ \\
\hline Boa satisfação com o serviço de saúde bucal & $22(75,9)$ & $19(82,6)$ & $41(78,8)$ \\
\hline $\begin{array}{l}\text { As orientações da equipe de saúde bucal atendem } \\
\text { às necessidades }\end{array}$ & $29(96,7)$ & $23(100)$ & $52(98,1)$ \\
\hline $\begin{array}{l}\text { Usuário considera o tempo de atendimento em } \\
\text { saúde bucal suficiente }\end{array}$ & $56(94,9)$ & $40(97,6)$ & $96(96,0)$ \\
\hline $\begin{array}{l}\text { O consultório odontológico possui boas } \\
\text { condições de limpeza }\end{array}$ & $57(96,6)$ & $42(100)$ & $99(98,0)$ \\
\hline $\begin{array}{l}\text { Os equipamentos odontológicos estão em boas } \\
\text { condições de uso }\end{array}$ & $54(91,5)$ & $39(92,9)$ & $93(92,1)$ \\
\hline
\end{tabular}

* Dados referentes ao módulo III da avaliação externa.

\section{DISCUSSÃO}

Analisou-se o perfil epidemiológico da assistência odontológica dos municípios da $4^{\mathrm{a}}$ Coordenadoria Regional de Saúde do Rio Grande do Sul (4 CRS/RS) a partir dos dados gerados no $2^{\mathrm{a}}$ ciclo do (PMAQ-AB), das 56 unidades analisadas a maior parte possui atendimento odontológico.

Apesar da maioria das UBS avaliadas possuírem atendimento odontológico com agendamento e capacidade de resolução dos casos, a maioria dos usuários não o utilizam. Este dado vai de acordo com os estudos que avaliaram o PMAQ em outras regiões brasileirascomo norte, nordeste e sudeste (9)(11)seeking to provide a better visibility of the organizational characteristics for its infrastructure, processes, and perception of its users. Material and Methods: modules I, II, and III of the database concerning to External Evaluation of the first cycle of the Brazilian National Program for Improving Access and Quality of Primary Care, carried out in 2012, were analyzed. Results: the health units of this study operated, mostly, in the morning and afternoon shifts $(88 \%$.O conceito de acesso, de modo geral, refere-se à percepção das necessidades de saúde, para que estas necessidades tornemse demandas, e, assim, possam se converter no uso de serviços de saúde (14). Por isso é necessário que, além da oferta de serviços odontológicos de qualidade, haja a percepção das necessidades de saúde por parte dos indivíduos. Nesse sentido, torna-se fundamental que a população disponha de informações que a possibilitem ampliar o conhecimento com relação a saúde e orientá-la na busca pelo auto cuidado(15)"container-title":"Ciência \& Saúde Coletiva","page":"3317-3329","volume" :"16","issue":"7",,"source":"'DOI.org (Crossref. Outro fator relevante pode estar relacionado a uma 
territorialização inadequada nos municípios, ou até mesmo inexistente, culminando na organização espacial da APS sem considerar as condições sociais e os limites territoriais, dificultando o uso dos serviços (16).

A maioria das unidades avaliadaspossui consultório odontológico e pelo menos um cirurgiãodentista, todavia, a cobertura odontológica da região Verdes Campos é baixa, sendo 36,8\% para o ano de 2019; já na região Entre Rios esta cobertura é de 69,7\% para o mesmo ano, apresentando um panorama mais favorável (17). Os altos custos advindos dos equipamentos e instrumentais que sãoessenciais para o atendimento odontológico, mesmo na atenção básica, podem ser um dos grandes entraves para a implantação de equipes de saúde bucal (1). Porém, é importante ressaltar que, mesmo considerando fundamental o aumento no número de equipes, este fato sozinho não é suficiente para melhorar os indicadores odontológicos, visto que o trabalho interno é tão relevante quanto a estrutura nesse processo (18).

Este estudou observou que a maioria das equipes de saúde bucal participam das reuniões de equipe, e que 63,6\% destas equipes recebem apoio de outros núcleos profissionais em casos complexos mostrando assim uma união multiprofissional e com a rede de atenção fundamental para aperfeiçoar o processo de trabalho.É importante que as equipes sejamcapazes de romper com o sistema hegemônico de atenção à saúde, este, centrado na assistência curativa, especializada e hospitalar, focado nos interesses econômicos e corporativistas. Portanto, é fundamental que as equipes sejam capazes de atuar conforme um sistema universal, capaz de valorizar a integralidade, a promoção de saúde e o cuidado humanizado (6)work process characteristics, and performance of a list of curative dental procedures by oral health teams. It involved an exploratory, cross-sectional study withmulticenter data collection from 11,374 oral health teams assessed by the National Program for Improvement of Access to and Quality of Primary Healthcare. Multilevel Poisson regression was used to obtain the prevalence of curative dental procedures, which was $69.51 \%$. The social/ economic and work variables that remained associated with the outcome included municipalities in which the proportion of primary care-sensitive admissions was below $28 \%$ and that of tooth extractions below $8 \%$; and oral health teams classified as type II (including oral health assistant and technician. A ruptura do modelo de atenção centrado na assistência curativa, depende da formação e da prática dos profissionais de saúde, sendo o investimento na formação de profissionais capazes de romper este conceito, tornando-se uma alternativa para melhorar os índices de eficiência obtidos pelas equipes de saúde da atenção primária(17).

A mudança na formação também pode gerar uma melhor articulação entre instituições de ensino e os serviços de saúde, fortalecendo o processo de educação continuada aos profissionais que já atuam no SUS(18)da prática do dia a dia de profissionais, usuários e gestores mostra-se fundamental para a resolução dos problemas encontrados na assistência à saúde e para a qualificação do cuidado prestado aos sujeitos. A mudança na formação acadêmica de estudantes e professores do campo da saúde também se tem mostrado necessária. Este artigo pretende fazer uma breve recuperação histórica dos marcos das propostas de educação na saúde para profissionais do Sistema Único de 
Saúde (SUS. Embora a maioria dos profissionais tenha relatado possuir formação complementar,é importante que seja formado um processo de construção de compromissos entre o serviço e a educação, fortalecendo assim a responsabilidade pública e a importância social das universidades com serviços de saúde, também trazendo medidas inovadoras para o contexto da educação em conjunto com as diretrizes do SUS, em especial a formação de profissionais capazes de valorizar a integralidade e a promoção de saúde, fugindo da lógica curativista, além de ser uma ferramenta essencial para a geração de educação continuada aos profissionais do SUS(18)da prática do dia a dia de profissionais, usuários e gestores mostra-se fundamental para a resolução dos problemas encontrados na assistência à saúde e para a qualificação do cuidado prestado aos sujeitos. A mudança na formação acadêmica de estudantes e professores do campo da saúde também se tem mostrado necessária. Este artigo pretende fazer uma breve recuperação histórica dos marcos das propostas de educação na saúde para profissionais do Sistema Único de Saúde (SUS.

Com relação ao planejamento e gestão da atenção básica, é importante que se considere a complexidade e a importância das diferentes áreas de conhecimento, uma vez que nenhum profissional consegue realizar seu trabalho sem a colaboração dos outros profissionais que compõem a equipe de saúde. Neste estudo, $75 \%$ das equipes relataram receber informações da gestão e $88 \%$ participam mensalmente de atividades de gestão e planejamento. Porém, além deste fato tambémé necessário que se considere as demandas propostas pelos usuários e comunidade, uma vez que tem como objetivo principal satisfazer as necessidades de saúde e intervir nos fatores de risco que possam provocar doenças e agravos à saúde (21).

Em relação à organização dos recursos humanos nos municípios brasileiros, é evidente que há problemas considerando que $8,9 \%$ das equipes de saúde bucal no Brasil apresentam apenas o cirurgião-dentista, ou seja, não há uma configuração de equipe de SBneste caso, e sim um trabalho isolado (22)based on information from the Basic Health Units with Oral Health and the municipalities to which these BHUs were implanted in Brazil in 2012. The collection was carried out through the use of Tablets, which contained an application with the instrument standardized and previously tested. The variables were: Oral Health Team, Macro region, Human Development Index, Population Coverage for OHT, Exodontic Ratio and Supervised Brushing. Data were analyzed in Stata 11 software. Analyzes of the absolute and relative frequencies of the studied variables were performed. Results: The prevalence of type II ESB was 9.9\% (95\% CI: 9.6-10.3. Portanto é necessário que os processos de captação de recursos humanos para as equipes de saúde bucal sejam repensados, propondo uma organização mais robusta, favorecendo a formação de equipes de saúde bucal compostas por no mínimo equipe do tipo I (20)based on information from the Basic Health Units with Oral Health and the municipalities to which these BHUs were implanted in Brazil in 2012. The collection was carried out through the use of Tablets, which contained an application with the instrument standardized and previously tested. The variables were: Oral Health Team, Macro region, Human Development Index, Population Coverage for OHT, Exodontic Ratio and Supervised Brushing. Data were analyzed in Stata 11 software. Analyzes of the absolute and relative frequencies of the studied variables were performed. Results: The prevalence of type II 
ESB was 9.9\% (95\% CI: 9.6-10.3.

Investimentos na qualificação da gestão ou gerência setorial são fatores necessários para a melhoria do acesso da população aos serviços de saúde bucal e da qualidade de atenção em saúde bucal no país. Além disso, e com base no contexto atual, é importante que seja crescente o número de estudos sobre a necessidade de acesso da população baseados em evidências cientificas, investigações que permitam garantir a alocação de recursos para a atenção básica de saúde e que permitam melhor quantificar e qualificar o impacto das intervenções e das políticas em saúde pública adotadas no Brasil(21).

O PMAQ-AB torna-se uma ferramenta importante na gestão dos recursos destinados à saúde, auxiliando na utilização dos serviços de saúde e contribuindo com melhorias no acesso e na qualidade, corroborando, assim, a tentativa de melhorar o desempenho dos indicadores de saúde. Entretanto, deve-se ressaltar que a incorporação de tais objetivos no processo de trabalho diário das equipes torna-se importante e necessária para que todo o serviço não fique condicionado a apenas um programa(22).

Com relação ao atendimento de gestantes, constatou-se que 90,9\% das equipes fazem atendimento e acompanham essas pacientes. É sabido que a gravidez provoca alterações no organismo da mulher, inclusive na cavidade bucal, acredita-se que toda mulher ao engravidar, deve ter o acompanhamento de uma equipe multidisciplinar, com a presença do cirurgião-dentista no grupo. Também é importante a orientação da equipe médica, considerando que a população de gestantes estaria melhor assistida na parte odontológica se o médico ginecologista-obstetra as orientasse e encaminhasse para a prevenção e o tratamento odontológico durante a gestação, cumprindo assim seu papel como promotor de saúde(23).

Quase a totalidade das equipes avaliadas realiza também atendimento as crianças com menos de cinco anos, achado positivo considerando o panorama do país, visto que apenas $22,1 \%$ das crianças brasileiras com essa idade já consultaram o dentista ao menos uma vez na vidasegundo a Pesquisa Nacional de Amostra por Domicílios (25). A atenção na primeira infância é uma conquista da odontologia, todavia, os dados com relação a cárie dentária nessa faixa etária indicam que a média foi de 2,43 dentes afetados (26). Estes dados epidemiológicos devem ser melhorados, o que pode ser atingido através de estratégias e ações de acesso e de promoção da saúde bucal que preconizem atendimentos preventivos em detrimento de curativos na atenção primária, e capazes modificar muitos dos fatores de risco das principais doenças bucais (27).

Há uma recomendação da Pesquisa Nacional de Saneamento Básico que a ocorra a organização e o desenvolvimento de ações de prevenção e controle do câncer bucal, que sejam capazes de identificar lesões bucais e que acompanhem e prestem assistência aos casos confirmados (24). É baixa a proporção de equipes que registram e acompanham os casos suspeitos de câncer bucal neste estudo, evidenciando a necessidade de qualificação das ações no sentido de garantir a integralidade 
do cuidado da saúde bucal da população. Sendo evidente a necessidade de capacitações e atualizações na área de diagnóstico clínico de lesões de boca aos cirurgiões dentistas(25).

É reconhecido pelo Ministério da Saúde que a assistência odontológica pública no Brasil se tem restringido quase que completamente aos serviços básicos, e propõe investimentos nos níveis secundário e terciário de atenção por meio dos Centros de Especialidades Odontológicas (CEO) (30), neste estudo constatou-se que somente $47,7 \%$ das equipes oferecem tratamento especializado. Outra dificuldade constatada pela pesquisa está atrelada à reabilitação protética pelas equipes de saúde bucal, pois $81,6 \%$ das equipes não oferecem este serviço. Avaliação do uso necessidade de prótese ajuda a entender o agravo 'edentulismo', servindo ao mesmo tempo para estimar a gravidade do problema e para subsidiar ações de planejamento (31)irrespective of their current socioeconomic position. Absolute and relative inequalities related to the use of dental prostheses were not related to childhood socioeconomic position. InCONCLUSIONS: These findings substantiate the association between life course socioeconomic circumstances and oral health in older adulthood, although use of dental prostheses was not related to childhood socioeconomic position. The study also highlights the long-lasting relation between childhood socioeconomic inequalities and oral health through the life course.

A avaliação positiva com relação ao atendimento e as condições do consultório odontológico vaiao encontro aos achados de outros estudos que consideram o ponto de vista do usuário (28)(29). Isso pode estar associado a adoção de condutas humanizadas, como o estabelecimento de vínculo entre o profissional e o usuário, o cuidado com a ambiência e a escuta qualificada (30). Todavia, esse dado deve ser analisado com prudência, visto que a satisfação pode estar relacionada a um baixo nível de expectativas por parte dos pacientes (31)bem como pela interpretação do atendimento recebido não como um direito, como de fato é, mas como uma doação ou indulgência (36).

Dentro das limitações presentes nesse estudo, podemos destacar que a seleção da amostra, além de ser apenas das equipes de saúde que aceitaram e demonstraram desejo de participar do programa, foi através dos usuários que buscavam atendimento na atenção primária, o que pode ter levado a um viés de seleção devido ao fato dessas pessoas serem mais acessíveis e possuírem algumas características comuns. Ademais, os participantes do estudo podem ter emitido parecer mais positivo na avaliação do serviço por estar sendo entrevistado dentro das dependências do mesmo, para evitar tensões e possíveis prejuízos na assistência recebida. Por fim, como se trata de um estudo observacional transversal descritivo, não é possível estabelecer associações tampouco relações causais. Porém como pontos fortes, foi o primeiro estudo realizado sobre serviços de saúde bucal com perspectiva regional abordando características estruturais, de processo de trabalho e de percepções dos usuários.

Foi possível identificar algumas diferenças sobre as características dos serviços de saúde bucal relacionadas com as regiões de saúde que compõe a $4^{\text {a }}$ Coordenadoria Regional de Saúde, principalmente em relação ao processo de trabalho das equipes de saúde bucal. Os resultados 
podem auxiliar no desenvolvimento de processos que tragam melhorias para as unidades, equipes e usuários da atenção básica no que tange o planejamento, organização e cuidado em saúde na $4^{\mathrm{a}} \mathrm{CRS} /$ RS. Este tipo de avaliação se mostra importante para o desenvolvimento de ações de saúde pública, uma vez que auxilia, os profissionais de saúde de outros locais do Brasil a organizarem a oferta dos serviços de saúde bucal para as necessidades da população contribuindo para a integralidade e a qualidade dos serviços de saúde bucal.

\section{REFERÊNCIAS BIBLIOGRÁFICAS}

1. Mattos GCM, Ferreira EF e, Leite ICG, Greco RM. A inclusão da equipe de saúde bucal na Estratégia Saúde da Família: entraves, avanços e desafios. Ciênc saúde coletiva. fevereiro de 2014;19(2):373-82.

2. Ribeiro CTM, Ribeiro MG, Araújo AP, Mello LR, Rubim L da C, Ferreira JES. O sistema público de saúde e as ações de reabilitação no Brasil. Rev Panam Salud Publica. julho de 2010;28(1):43-8.

3. Reis WG, Scherer MD dos A, Carcereri DL. O trabalho do Cirurgião-Dentista na Atenção Primária à Saúde: entre o prescrito e o real. Saúde debate. março de 2015;39(104):56-64.

4. Pinto HA, Sousa ANA de, Ferla AA. O Programa Nacional de Melhoria do Acesso e da Qualidade da Atenção Básica: faces de uma política inovadora. Saúde em Debate [Internet]. 2014 [citado 14 de agosto de 2019];38(special). Disponível em: http://www.gnresearch.org/ doi/10.5935/0103-1104.2014S027

5. Seidl HMF, Vieira S de P, Fausto MCR, Lima R de CD, Gagno JL. Gestão do trabalho na atenção básica em saúde: uma análise a partir da perspectiva das equipes participantes do PMAQ2012. Saúde em Debate [Internet]. 2014 [citado 15 de agosto de 2019];38(special). Disponível em: http:/www.gnresearch.org/doi/10.5935/0103-1104.2014S008

6. Neves M, Giordani JM do A, Hugo FN. Atenção primária à saúde bucal no Brasil: processo de trabalho das equipes de saúde bucal. Ciênc saúde coletiva. maio de 2019;24(5):180920.

7. Tanaka OY, Tamaki EM. O papel da avaliação para a tomada de decisão na gestão de serviços de saúde. Ciêncsaúdecoletiva. abril de 2012;17(4):821-8.

8. Donabedian A. Evaluating the Quality of Medical Care. :39.

9. Casotti E, Contarato PC, Fonseca ABM, Borges PK de O, Baldani MH. Atenção em saúde bucal no Brasil: reflexões a partir da avaliação externa do PMAQ-AB. Saúde em Debate [Internet]. 2014 [citado 14 de agosto de 2019];38(special). Disponível em: http://www.gnresearch. 
org/doi/10.5935/0103-1104.2014S011

10. Pereira APF. UNIVERSIDADE FEDERAL DE SÃO CARLOS CENTRO DE CIÊNCIAS BIOLÓGICAS E DA SAÚDE PROGRAMA DE PÓS-GRADUAÇÃO EM GESTÃO DA CLÍNICA MESTRADO PROFISSIONAL EM GESTÃO DO TRABALHO. 2017;105.

11. Hirooka LB, Catanante GV, Porto HS, Caccia-Bava M do CGG. Organização da saúde bucal em uma região do estado de São Paulo segundo a Avaliação Externa do PMAQ-AB, 2012. Rev Bras Odontol. 29 de junho de 2017;74(2):101.

12. Moreira R da S, Nico LS, Tomita NE, Ruiz T. A saúde bucal do idoso brasileiro: revisão sistemática sobre o quadro epidemiológico e acesso aos serviços de saúde bucal. Cad Saúde Pública. dezembro de 2005;21(6):1665-75.

13. Schio B. UNIVERSIDADE FEDERAL DE SANTA MARIA. 2019;30.

14. Pinheiro RS, Torres TZG de. Uso de serviços odontológicos entre os Estados do Brasil. Ciênc saúde coletiva. dezembro de 2006;11(4):999-1010.

15. Haikal DS, Paula AMB de, Martins AME de BL, Moreira AN, Ferreira EF e. Autopercepção da saúde bucal e impacto na qualidade de vida do idoso: uma abordagem quantiqualitativa. Ciênc saúde coletiva. julho de 2011;16(7):3317-29.

16. Faria R. A TERRITORIALIZAÇÃO DA ATENÇÃO PRIMÁRIA À SAÚDE DO SUS: AVALIAÇÃO DOS RESULTADOS PRÁTICOS IMPLEMENTADOS NUMA CIDADE DA REGIÃO SUL DO BRASIL. Hygeia [Internet]. 9 de outubro de 2018 [citado 15 de agosto de 2019]; Disponível em: http://www.seer.ufu.br/index.php/hygeia/article/view/44129

17. Rocha TAH, Silva NC da, Barbosa ACQ, Rodrigues JM. Human Resource Management in Health and Performance of Work Process in the Primary Health Care-An Efficiency Analysis in a Brazilian Municipality. Journal of Health Management. setembro de 2014;16(3):365-79.

18. Batista KBC, Gonçalves OSJ. Formação dos profissionais de saúde para o SUS: significado e cuidado. Saude soc. dezembro de 2011;20(4):884-99.

19. Bertusso FR, Rizzotto MLF. PMAQ na visão de trabalhadores que participaram do programa em Região de Saúde do Paraná. Saúde debate. junho de 2018;42(117):408-19.

20. Martins PHS, Amaral Júnior, OL, Faustino-Silva, DD, Torres, Luisa HN, Giordani JM do A, Unfer, B. Desigualdades na distribuição das equipes de saúde bucal no Brasil. 2017;23:10.

21. Campos RTO, Ferrer AL, Gama CAP da, Campos GW de S, Trapé TL, Dantas DV. Avaliação da qualidade do acesso na atenção primária de uma grande cidade brasileira na 
perspectiva dos usuarios. Saúde em Debate [Internet]. 2014 [citado 17 de setembro de 2019];38(special). Disponível em: http://www.gnresearch.org/doi/10.5935/0103-1104.2014S019

22. Medrado JRS, Casanova AO, Oliveira CCM de. Estudo avaliativo do processo de trabalho das Equipes de Atenção Básica a partir do PMAQ-AB. Saúde debate. dezembro de 2015;39(107):1033-43.

23. Amadei SU. Prescrição medicamentosa no tratamento odontológico de grávidas e lactantes. Rev Gaúcha Odontol. 2011;7.

24. Bulgareli JV, Diniz OCCF, Faria ET de, Vazquez F de L, Cortellazzi KL, Pereira AC. Prevenção e detecção do câncer bucal: planejamento participativo como estratégia para ampliação da cobertura populacional em idosos. Ciênc saúde coletiva. dezembro de 2013;18(12):3461-73.

25. Casotti E, Monteiro ABF, Castro Filho EL de, Santos MP dos. Organização dos serviços públicos de saúde bucal para diagnóstico precoce de desordens com potencial de malignização do estado do Rio de Janeiro, Brasil. Ciênc saúde coletiva. maio de 2016;21(5):1573-82.

26. Aquilante AG, Aciole GG. Oral health care after the National Policy on Oral Health “Smiling Brazil”: a case study. Ciênc saúde coletiva. janeiro de 2015;20(1):239-48.

27. Andrade FB de, Antunes JLF, Souza Junior PRB de, Lima-Costa MF, Oliveira CD. Life course socioeconomic inequalities and oral health status in later life. Rev saúde pública. 24 de janeiro de 2019;52(Suppl 2):7s.

28. Santiago RF, Mendes A da CG, Miranda GMD, Duarte PO, Furtado BMASM, Souza WV de. Qualidade do atendimento nas Unidades de Saúde da Família no município de Recife: a percepção do usuários. Ciênc saúde coletiva. janeiro de 2013;18(1):35-44.

29. Moimaz SAS, Lima AMC, Garbin CAS, Corrente JE, Saliba NA. Avaliação do usuário sobre o atendimento odontológico no Sistema Único de Saúde: uma abordagem à luz da humanização. Ciênc saúde coletiva. dezembro de 2016;21(12):3879-87.

30. Lima CC, Guzman SM, Benedetto MACD, Gallian DMC. Humanidades e humanização em saúde: a literatura como elemento humanizador para graduandos da área da saúde. Interface (Botucatu). 2014;18(48):139-50.

31. Brandão AL da RB da S, Giovanella L, Campos CEA. Avaliação da atenção básica pela perspectiva dos usuários: adaptação do instrumento EUROPEP para grandes centros urbanos brasileiros. Ciênc saúde coletiva. janeiro de 2013;18(1):103-14. 
$159 / /$

32. Traverso-Yépez M, Morais NA de. Reivindicando a subjetividade dos usuários da Rede Básica de Saúde: para uma humanização do atendimento. Cad Saúde Pública. fevereiro de 2004;20(1):80-8.

Artigo apresentado em setembro de 2019 Artigo aprovado janeiro de 2020 Artigo publicado em maio de 2020 